\title{
Advances in Cryo-Electron Microscopy for Understanding Energy Materials
}

Danielle Markovich $^{1}$, Michael Zachman ${ }^{2}$, Seun-Ho Yu ${ }^{1}$, Ryan Selhorst ${ }^{3}$, Taylor Moon ${ }^{1}$, Héctor Abruña ${ }^{1}$, Kevin Noonan ${ }^{3}$ and Lena Kourkoutis ${ }^{1}$

${ }^{1}$ Cornell University, Ithaca, New York, United States, ${ }^{2}$ Oak Ridge National Laboratory, Oak Ridge, Tennessee, United States, ${ }^{3}$ Carnegie Mellon University, Pittsburgh, Pennsylvania, United States

Many modern energy devices rely on highly reactive materials, solid-liquid interfaces, or both, for their operation and performance. Physical and chemical processes at complex interfaces between dissimilar materials, for example, play a crucial role in catalysis, solar energy and fuel generation, and electrochemical energy storage, however, such interfaces are notoriously difficult to characterize at high spatial resolution in their native environment [1]. Recent advances in cryo-focused ion beam milling (cryoFIB) coupled with cryo-scanning transmission electron microscopy (cryo-STEM) have enabled nanometer scale characterization of processes at complex solid-liquid interfaces stabilized by rapid-freezing [2, 3]. Samples containing highly reactive materials, such as alkali metals, present additional challenges for characterization since exposed regions react quickly with air during transfer into a microscope or with contaminants in the vacuum during characterization, altering the sample's surface chemistry [4]. Cryogenic cooling has been shown on freeze out these reactions allowing structural characterization by cryo-TEM and cryo-STEM $[3,5,6]$.

In this talk, we will first focus on recent developments that have enable atomic resolution STEM imaging and spectroscopy of energy materials and their interfaces at cryogenic temperatures. Technical advances in spectroscopic imaging using electron energy loss spectroscopy (EELS) have allowed a material's chemical and electronic structure to be resolved at the atomic scale. While this can now be achieved almost routinely for radiation-hard materials studied at room temperature, cryogenic applications are hampered by the reduced stability of side-entry cryo-holders [7]. This is particularly challenging for spectroscopic mapping as it requires pixel dwell times that are orders of magnitude larger than typical imaging dwell times. Using a Gatan K2 direct electron detector enabled for spectroscopy with improved detector quantum efficiency, point spread function, and signal-to-noise ratio compared to indirect, scintillatorbased detectors, we demonstrate atom-resolved spectroscopic mapping at liquid nitrogen temperature [8]. Operating at cryogenic temperatures not only allows imaging of reactive materials such as alkali metals but also enables the study of nanostructures snap-frozen in solution and suppresses carbon built-up during imaging which is particularly important for nanoparticles that are not robust to standard cleaning methods.

To demonstrate the benefit of cryo-STEM for understanding electrochemical processes, we will discuss the lithiation process of a single $\mathrm{MoS}_{2}$ monolayer. Here, cryogenic sample preparation is required to suppress unwanted reactions prior to imaging (Fig. 1a). Transfer into the microscope under liquid nitrogen and cooling during the experiment ensures that the sample remains pristine. Atomic-resolution and elemental contrast in STEM allows us to identify that monolayers can undergo a conversion reaction even without a substrate during lithiation in a liquid electrolyte (Fig. 1). Bilayer $\mathrm{MoS}_{2}$ on the other hand undergoes an intercalation reaction before conversion to Mo and $\mathrm{Li}_{2} \mathrm{~S}$ nanoparticles [9]. The cryo-STEM imaging stability demonstrated here, have allowed us to gain unique atomic-scale insights into the lithiation processes 2D layered materials down to the monolayer limit. Such insights afforded by cryogenic electron microscopy will enable the design of improved materials for energy applications. 
In monolayer systems projection imaging is sufficient to understand many of the fundamental processes. However, many energy materials are inherently three dimensional. One example is a alkaline anion exchange membrane which plays an important role in a range of energy systems such as fuel cells, electrolyzers and redox flow batteries. We will show how cryo-STEM, cryo-TEM and spectroscopy can reveal the tight connection between 3D architecture of such polymer membranes (Fig. 2) and its performance. [10]

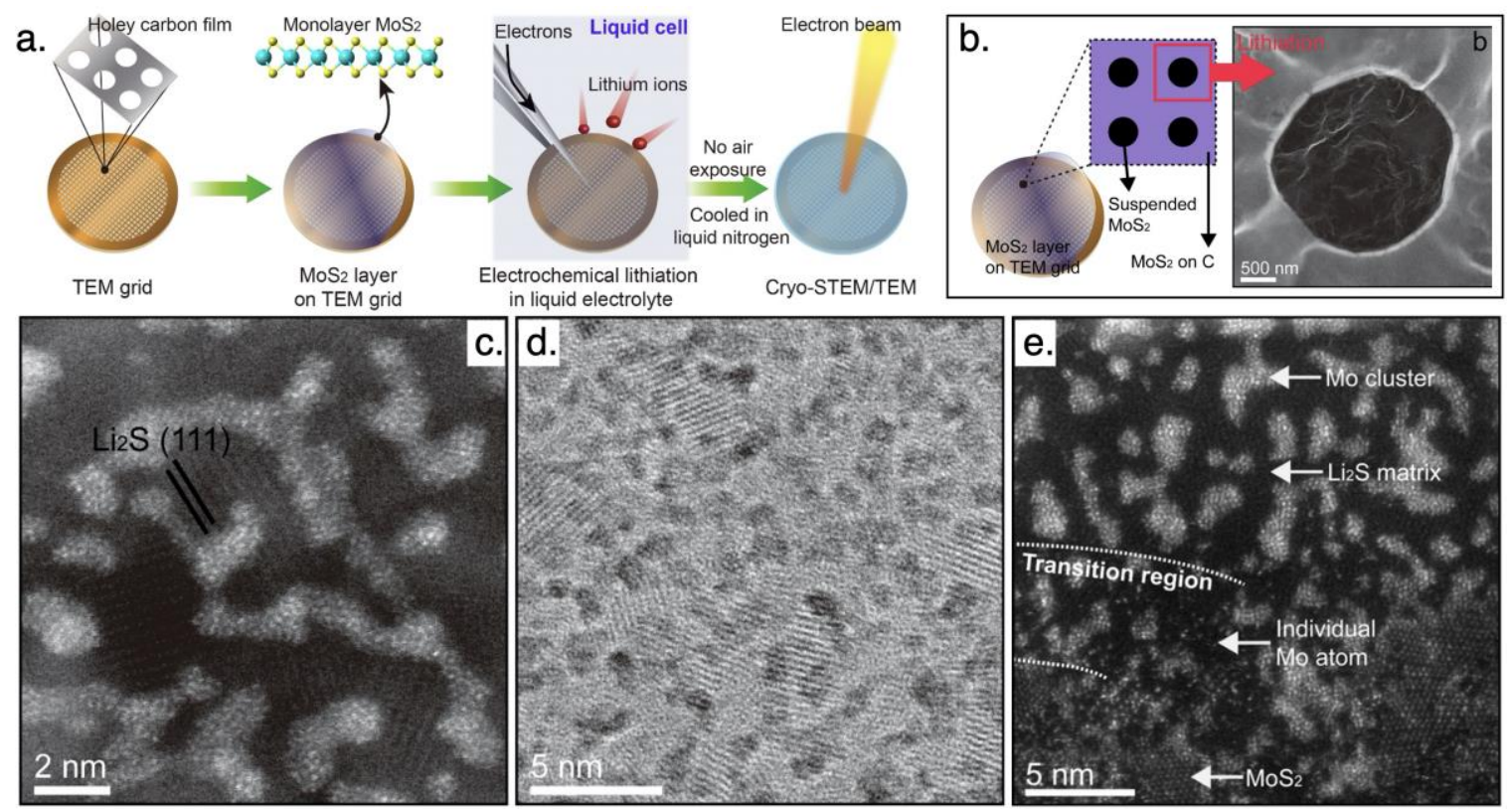

Figure 1. Atomic-resolution cryo-S/TEM of MoS2 lithiation. (a) Schematic illustration of the electrochemical lithiation and cryo-EM characterization of a MoS2 layer supported on a holey carbon coated TEM grid. (b) Lithiation of a suspended MoS2 monolayer. (c) Atomic-resolution annular darkfield cryo-STEM and (d) cryo-TEM image of a fully lithiated MoS2 monolayer confirming the presence of Mo metal and Li2S nanoparticles after lithiation. (e) Cryo-STEM of MoS2 area that is only partially lithiated [adapted from Ref. 9].
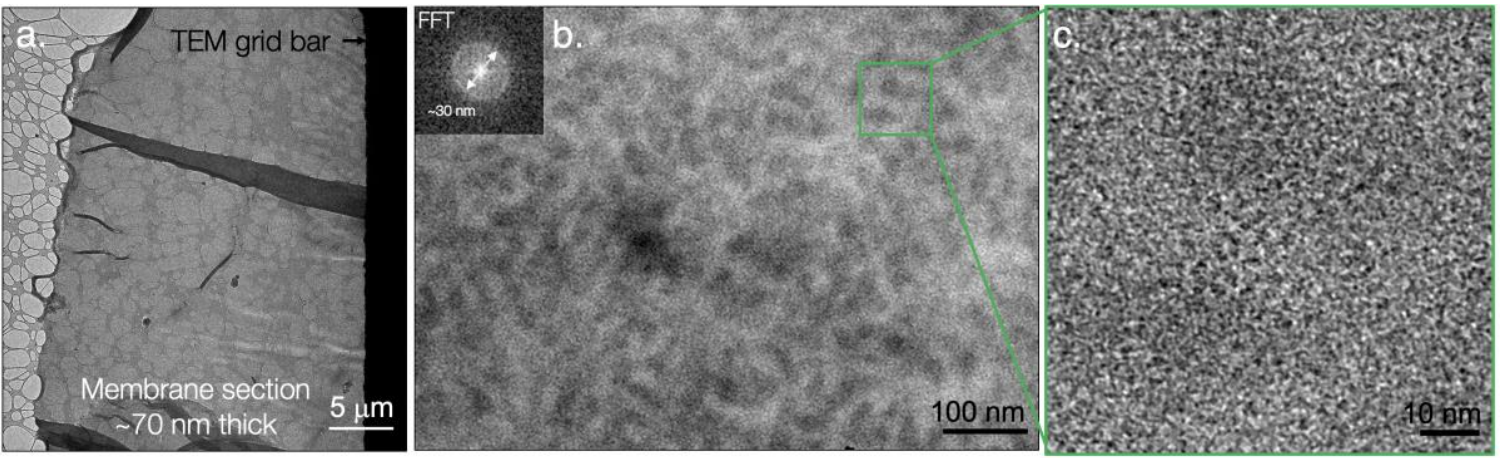

Figure 2. Cryo-TEM imaging of alkaline anion exchange membranes provides direct picture of nanoscale ordering. (a) Cryo-ultramicrotomy allows preparation of relatively uniform, thin sections of polymer membranes. (b) Cryo-TEM of unstained membranes reveals nanoscale ordering, i.e., microphase separation of hydrophilic and hydrophobic domains. Contrast arises from heavy cation incorporation during synthesis. No additional staining is required which avoids artifacts during imaging. (c) Imaging with a K3 direct electron detector captures both microphase separation with an ordering length scale of 
$\sim 30 \mathrm{~nm}$ (b, inset FFT) as well as $\sim 1 \mathrm{~nm}$ pore structure in the membrane giving rise to hydroxide conductivity.

\section{References}

[1] F. Zaera, Chem. Rev. 112 (2012), p. 2920.

[2] M. J. Zachman, et al., Microsc. Microanal. 22 (2016), p. 1338.

[3] M.J. Zachman, et al., Nature 560 (2018), p. 345.

[4] D-R. Liu and D. B. Williams, Philos. Mag. B 53 (1986), p. L123.

[5] Y. Li, et al., Science 358 (2017), p. 506.

[6] X. Wang, et al., Nano Lett. 17 (2017), p. 7606.

[7] Goodge, et al., arXiv:2001.11581.

[8] Goodge, et al. Microsc. Microanal. 24 S1 (2018), p. 1844-1845.

[9] S-H. Yu, et al., Adv. Energy Mater. 9 (2019) 1902773.

[10] This work is supported in part by NSF (DMR-1654596, DMR-1429155, DMR-1719875) and the Center for Alkaline Based Energy Solutions (CABES) funded by DOE (DE-SC0019445). 
https://doi.org/10.1017/S1431927620018838 Published online by Cambridge University Press 